\title{
Le bloc de branche gauche instable et provocable
}

\section{Lausanne}

Les images en bloc de branche droit ou gauche sont presque toujours permanentes. Les travaux minutieux de Lenègre ont dé-finitivement établi que ces images correspondent à des lesions destructives de la branche impotente.

11 existe néanmoins des cas de bloc de branche instables, ou intermittents, ou éphémères, ou encore : à repetition. On en rencontre dans $\Gamma$ infarctus antérieur pour le bloc de branche droite et j'en donne un exemple très original avec bloc de branche droite initial pendant les 4 premiers jours, et récidive 2 ans $1 / 8$ plus tard, pendant quelques jours, à la suite d'une chute sur Гabdomen. Pour le bloc de branche gauche, les conditions semblent assez différentes et j'ai recueilli depuis 7 ans une douzaine de cas dans lesquels le bloc de branche gauche est lie à $\Gamma$ insuffisance ventriculaire gauche et à $\Gamma$ insuffisance coronaire, ou simplement à Thyper-tension artérielle. Bischoff nous en avait montré un exemple, il y a 2 ans. Dans ces exemples le bloc de branche gauche disparait avec le repos et le traitement, mais alors il reste provocable à volonté par un tout petit effort.

Dans la pathogénie de ce bloc de branche gauche instable et provocable, il faut faire une place aux hémorragies septales sous-endocardiques si bien décrites à Zurich par Berthe Capt en 1948. A signaler encore le bon effet de Гoxygénothérapie décrit par Joseph Levy en 1950, ce qui confirme le role de Tanoxémie dans ce trouble de conduction original. 